\title{
Protecting Frontline Health Care Workers from COVID-19 with Hydroxychloroquine Pre-exposure Prophylaxis: A structured summary of a study protocol for a rando- mised placebo-controlled multisite trial in Toronto, Canada
}

Julie K. Wright ${ }^{1,2,3,4,5}$, Darrell H. S. Tan 1,6,7,8,9, Sharon L. Walmsley ${ }^{1,6,10}$, Jennifer Hulme ${ }^{11,12}$, Erin O'Connor ${ }^{12,13}$, Carolyn Snider ${ }^{9,13,14}$, Ivy Cheng ${ }^{13,15}$, Adrienne K. Chan ${ }^{1,6,16}$, Bjug Borgundvaag ${ }^{11,17}$, Shelley McLeod ${ }^{11,17}$, Michael H. Gollob ${ }^{18}$, Rosemarie J. Clarke ${ }^{10}$, Linda Dresser ${ }^{19,20}$, Fatima Haji ${ }^{21}$, Tony Mazzulli1 ${ }^{1,4,422}$, Samira Mubareka1,3,16,23, Peter Jüni ${ }^{6,24,25}$, Dominic Lee ${ }^{25}$, George Tomlinson ${ }^{6,26}$, Kevin C. Kain ${ }^{1,3,4,5 \dagger}$ and Megan Landes ${ }^{11,12^{*}+}$ (D)

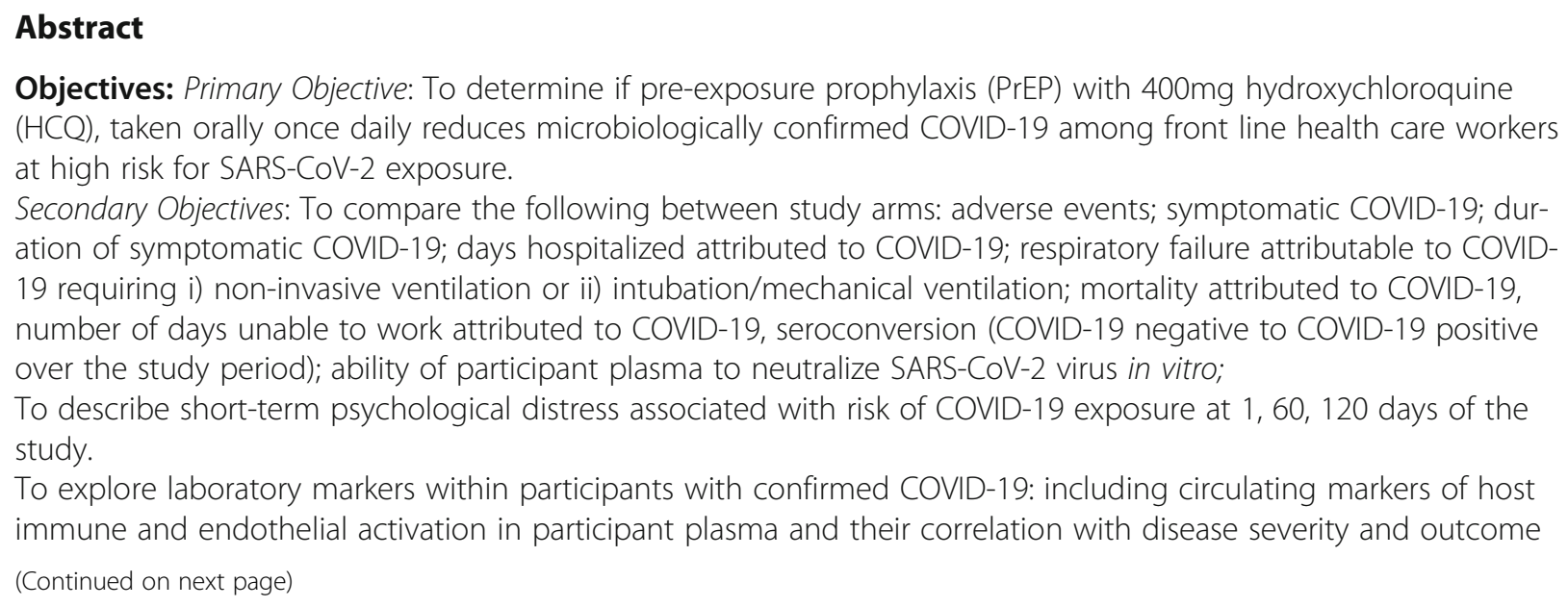

\footnotetext{
*Correspondence: megan.landes@uhn.ca

${ }^{\dagger}$ Kevin C. Kain and Megan Landes are Co-senior authors

${ }^{11}$ Divisions of Emergency Medicine, Department of Family and Community

Medicine, University of Toronto, Toronto, Ontario, Canada

${ }^{12}$ Emergency Department, University Health Network, Toronto, Ontario,

Canada

Full list of author information is available at the end of the article
}

(c) The Author(s). 2020 Open Access This article is licensed under a Creative Commons Attribution 4.0 International License, which permits use, sharing, adaptation, distribution and reproduction in any medium or format, as long as you give appropriate credit to the original author(s) and the source, provide a link to the Creative Commons licence, and indicate if changes were made. The images or other third party material in this article are included in the article's Creative Commons licence, unless indicated otherwise in a credit line to the material. If material is not included in the article's Creative Commons licence and your intended use is not permitted by statutory regulation or exceeds the permitted use, you will need to obtain permission directly from the copyright holder. To view a copy of this licence, visit http://creativecommons.org/licenses/by/4.0/ The Creative Commons Public Domain Dedication waiver (http://creativecommons.org/publicdomain/zero/1.0/) applies to the data made available in this article, unless otherwise stated in a credit line to the data. 
(Continued from previous page)

Trial design: The HEROS study is a two-arm, parallel-group, individually randomized (1:1 allocation ratio), placebo controlled, participant and investigator-blinded, multi-site superiority trial of oral HCQ 400 mg taken once daily for 90 days as PrEP to prevent COVID-19 in health care workers at high risk of SARS-CoV-2 exposure. At 90 days, there is an open label extension wherein all participants are offered a one-month course of HCQ 400mg once daily for PrEP of COVID-19.

Participants: Frontline HCWs aged 18 years of age or older, at high risk of SARS-CoV-2 exposure (including staff of emergency departments, intensive care units, intubation teams, COVID-wards, and staff deployed to Long Term Care facilities) of five academic hospitals in downtown Toronto, Canada.

Exclusion criteria include: currently pregnant, planning to become pregnant during the study period, and/or breast feeding; known hypersensitivity/allergy to hydroxychloroquine or to 4-aminoquinoline compounds; current use of hydroxychloroquine; known prolonged QT syndrome and/or baseline resting ECG with QTc>450 ms and/or concomitant medications which simultaneously may prolong the QTc that cannot be temporarily suspended/replaced; known pre-existing retinopathy, G6PD deficiency, porphyria, liver disease including cirrhosis, encephalopathy, hepatitis or alcoholism, diabetes on oral hypoglycemics or insulin, or renal insufficiency/failure; disclosure of selfadministered use of hydroxychloroquine or chloroquine within 12 weeks prior to study; confirmed symptomatic COVID-19 at time of enrollment.

Intervention and comparator: Intervention: hydroxychloroquine, 400mg (2 tablets) orally per day. Comparator: placebo, two tablets visually identical to the intervention, orally per day

Main outcomes: The primary outcome is microbiologically confirmed COVID-19 (i.e. SARS-CoV-2 infection). This is a composite endpoint which includes positive results from any validated SARS-CoV-2 diagnostic assay including detection of viral RNA, and/or seroconversion.

Participants will be assessed at baseline, and then undergo monthly follow-up at day 30, 60, and 90, 120. At each visit, participants will provide an oropharyngeal sample, blood sample, and will undergo electrocardiogram monitoring of the QTC interval.

Secondary outcome measures include: adverse events; symptom duration of COVID-19; days of hospitalization attributed to COVID-19; respiratory failure requiring ventilator support attributed to COVID-19; mortality attributed to COVID-19; total days off work attributed to COVID-19; seropositivity (reactive serology by day 120); and short term psychological impact of exposure to SARS-CoV-2 at day 1, 60, 120 days using the K10, a validated measure of nonspecific psychological distress.

Randomisation: Within each site, participants will be individually randomized to either the intervention arm with HCQ or the placebo arm using a fixed 1:1 allocation ratio using an interactive web-based response system to ensure concealment of allocation. Randomization schedules will be computer-generated and blocked using variable block sizes.

Blinding (masking): All participants, research coordinators, technicians, clinicians and investigators will be blinded to the participant allocation group.

Numbers to be randomised (sample size) N=988, randomised into two groups of 494 patients.

Trial Status: This summary describes protocol version No. 1.6, May 15, 2020. Recruitment is ongoing - started April 20, 2020 and anticipated end date is July 30, 2021

Trial registration: ISRCTN.com Identifier: ISRCTN14326006, registered April 14, 2020.

Full protocol: The full protocol is attached as an additional file, accessible from the Trials website (Additional file 1). In the interest in expediting dissemination of this material, the familiar formatting has been eliminated; this Letter serves as a summary of the key elements of the full protocol.

The study protocol has been reported in accordance with the Standard Protocol Items: Recommendations for Clinical Interventional Trials (SPIRIT) guidelines (Additional file 2).

Keywords: COVID-19, Randomised placebo controlled trial, protocol, hydroxychloroquine, viral PCR, serology, psychological distress 


\section{Supplementary information}

Supplementary information accompanies this paper at https://doi.org/10. 1186/s13063-020-04577-8.

\section{Additional file 1.}

Additional file 2.

\section{Acknowledgements}

We would like to acknowledge Elayna Fremes, Alexandra McKnight, Karima Noordin and Danny Marchese for their assistance in rapidly enabling the setup of this study.

\section{Authors' contributions}

$\mathrm{ML}$ and KK are the co-Principal Investigators and Study Sponsors. JKW, ML, KCK, DHST and SLW conceived and designed the trial, and prepared the study protocols for REB review. GT directed the statistical analysis plan. The Applied Health Research Centre of St. Michael's Hospital (AHRC) (PJ and DK) developed the data management plan and study database. TM is the lead microbiologist responsible for sample processing and SARS-CoV-2 PCR. SM will lead the virus neutralization studies. RC is the Project Manager. MG is the cardiology consultant. SLW/JH/EO (University Health Network), DHST/CS (St Michael's/Unity Health), IC/AC (Sunnybrook Health Sciences Center), BB/SM (Sinai Health System), serve as the Study Site leads and implemented the study protocols at their respective institutions. LD and FH are the study pharmacists.

\section{Funding}

The HEROS trial is funded by: The National Research Council of Canada, The Thistledown Foundation, The GeoSentinel Foundation, The Slaight Family Foundation University Health Network Foundation, and anonymous donation. APOTEX donated the study drug and placebo. JKW is supported by a Vanier Scholarship (CIHR) and the Eliot-Phillipson Clinician Scientist Training Program (Department of Medicine, University of Toronto). DHST is supported by a Tier 2 Canada Research Chair in HIV Prevention and STIs. SLW receives career support from the Ontario HIV treatment network as Chair in HIV Clinical Management and Aging PJ is supported by a Tier 1 Canada Research Chair in Clinical Epidemiology of Chronic Diseases. KCK is supported by a Tier 1 Canada Research Chair in Molecular Parasitology and a CIHR Foundation grant FDN-148439.

The funding bodies have had / will have no role in the design of the study, data collection, analysis, interpretation, or dissemination of study findings to either journals, general public or scientific meetings.

\section{Availability of data and materials}

Data will be made available from the author on reasonable request. Please contact Dr. Megan Landes (corresponding author).

\section{Ethics approval and consent to participate}

Ethical approval was granted by Clinical Trials Ontario (CTO) (Project ID: 2132). Initial approval was granted 8 April 2020. The Board of Record is the University Health Network (UHN) Research Ethics Board (REB). The public listing of the study can be found here: https://www.ctontario.ca/covid-19 clinical-trial-resources/ The HEROS trial received ethical approval from an appropriate ethical committee as described above. This study will be conducted in accordance with the ICH-GCP Guidelines and the principles in the Declaration of Helsinki. Potential participants are invited to view an online information video outlining the risks, benefits and procedures of the trial, and then will be invited to provide written informed consent prior to being enrolled.

\section{Consent for publication}

Not applicable.

\section{Competing interests}

The authors declare that they do not have any competing interests.

\section{Author details}

'Division of Infectious Diseases, Department of Medicine, University of Toronto, Toronto, Ontario, Canada. ${ }^{2}$ Eliot Phillipson Clinician Scientist Training Program, Department of Medicine, University of Toronto, Toronto, Ontario,
Canada. ${ }^{3}$ Department of Laboratory Medicine and Pathobiology, University of Toronto, Toronto, Ontario, Canada. ${ }^{4}$ Division of Infectious Diseases, Department of Medicine, University Health Network/Sinai Health System, Toronto, Ontario, Canada. ${ }^{5}$ Sandra Rotman Laboratories, Sandra Rotman Centre for Global Health, UHN-Toronto General Hospital, Toronto, Ontario, Canada. ${ }^{6}$ Institute of Health Policy, Management and Evaluation, University of Toronto, Toronto, Ontario, Canada. ${ }^{7}$ Division of Infectious Diseases, Department of Medicine, St. Michael's Hospital, Toronto, Ontario, Canada. ${ }^{8}$ MAP Centre for Urban Health Solutions, St Michaels Hospital, Toronto, Ontario, Canada. ' $\mathrm{Li}$ Ka Shing Knowledge Institute, St. Michael's Hospital, Toronto, Ontario, Canada. ${ }^{10}$ Immunodeficiency Clinic, Toronto General Hospital, University Health Network, Toronto, Ontario, Canada. ${ }^{11}$ Divisions of Emergency Medicine, Department of Family and Community Medicine, University of Toronto, Toronto, Ontario, Canada. ${ }^{12}$ Emergency Department, University Health Network, Toronto, Ontario, Canada. ${ }^{13}$ Divisions of Emergency Medicine, Department of Medicine, University of Toronto, Toronto, Ontario, Canada. ${ }^{14}$ Emergency Department, St. Michael's Hospital, Toronto, Ontario, Canada. ${ }^{15}$ Emergency Department, Sunnybrook Health Sciences Centre, Toronto, Ontario, Canada. ${ }^{16}$ Division of Infectious Diseases, Sunnybrook Health Sciences Centre, Toronto, Ontario, Canada. ${ }^{17}$ Schwartz/ Reisman Emergency Medicine Institute, Sinai Health, Toronto, Ontario, Canada. ${ }^{18}$ Division of Cardiology, Department of Medicine, University Health Network, Toronto, Ontario, Canada. ${ }^{19}$ Leslie Dan Faculty of Pharmacy, University of Toronto, Toronto, Ontario, Canada. ${ }^{20}$ Antimicrobial Stewardship Program, Sinai Health System/University Health Network, Toronto, Ontario, Canada. ${ }^{21}$ Investigational Pharmacy Services, University Health Network, Toronto, Ontario, Canada. ${ }^{22}$ Department of Microbiology, University Health Network / Sinai Health System, Toronto, Ontario, Canada. ${ }^{23}$ Sunnybrook Research Institute, Sunnybrook Health Sciences Centre, Toronto, Ontario, Canada. ${ }^{24}$ Department of Medicine, University of Toronto, Toronto, Ontario, Canada. ${ }^{25}$ Applied Health Research Centre (AHRC), St. Michael's Hospital, Toronto, Ontario, Canada. ${ }^{26}$ Department of Medicine, University Health Network, Toronto, Ontario, Canada.

Received: 29 June 2020 Accepted: 2 July 2020 Published online: 14 July 2020

\section{Publisher's Note}

Springer Nature remains neutral with regard to jurisdictional claims in published maps and institutional affiliations.

Ready to submit your research? Choose BMC and benefit from:

- fast, convenient online submission

- thorough peer review by experienced researchers in your field

- rapid publication on acceptance

- support for research data, including large and complex data types

- gold Open Access which fosters wider collaboration and increased citations

- maximum visibility for your research: over $100 \mathrm{M}$ website views per year

At BMC, research is always in progress.

Learn more biomedcentral.com/submissions 